\title{
El "giro teológico" de la fenomenología: Introducción al debate
}

\author{
Carlos Enrique Restrepo*
}

\begin{abstract}
Resumen: El polémico libro de Dominique Janicaud sobre El giro teológico de la fenomenología francesa ha puesto a pensadores como E. Lévinas, J.-L. Marion, M. Henry y J.-L. Chrétien en el centro de los debates contemporáneos. Con veinte años de discusión desde esta publicación, el debate ha ido dejando su marca en el contexto latinoamericano. Este ensayo hace un balance del estado de la discusión en nuestro contexto, ofreciendo un inventario de textos orientado a servir de guía e introducción al debate.
\end{abstract}

Palabras clave: Fenomenología, "giro teológico", filosofía francesa, contexto latinoamericano, fenómeno saturado.

Abstract: Dominique Janicaud's polemic book on The Theological Turn of French Phenomenology has placed thinkers such as E. Lévinas, J.-L. Marion, M. Henry and J.-L. Chrétien at the center of the contemporary debate. With twenty years of discussion since the book was first published, the debate has left its mark on the Latin American context. This paper takes stock of the discussion in our context, offering an inventory of writings intended to serve as a guide and introduction to the debate.

Key words: Phenomenology, "theological turn", french Philosophy, Latin American context, saturated phenomenon.

Résumé: Le livre de Dominique Janicaud sur Le tournant théologique de la phénoménologie française a mis penseurs tels que E. Levinas, J.-L. Marion, M. Henry et J.-L. Chrétien au coeur des débats contemporains. Après vingt ans de discussion depuis cette publication, le débat a commencé à laisser des traces dans le contexte latino-américain. Cet article fait le bilan de l'état de la discussion dans notre contexte et offre un inventaire de textes qui serviront de guide ainsi que d'introduction au débat.

Mots-clés: Phénoménologie, "tournant théologique", philosophie française, contexte latinoaméricain, phénomène saturé.

* Instituto de Filosofía, Universidad de Antioquia, Medellín, Colombia. (alteridad@quimbaya.udea.edu.co).

Recibido: 2010 - 10 - 15

Aprobado: 2010 - 11 - 18 
En 1991, el filósofo francés Dominique Janicaud publicó en París un pequeño libro de escasas noventa páginas que dio la pauta para uno de los más importantes debates filosóficos contemporáneos: El giro teológico de la fenomenología francesa ${ }^{1}$. Con este título, el autor enfilaba sus críticas contra algunos filósofos del contexto francés que, solapados - según él- en la "ciencia estricta" proyectada por Husserl, habrían efectuado un inusitado retorno hacia conceptos y cuestiones de cuño teológico, en lo que resultaba ser una ampliación abusiva del título "fenomenología". Leídos bajo la influencia de Heidegger, los nombres de Emmanuel Lévinas, Jean-Luc Marion y Michel Henry son los principales blancos hacia los que se dirige el ataque. El curso del debate, prolongado por Janicaud en La phénoménologie éclatée ${ }^{2}$, y por muchos otros autores en numerosos escritos, implicará también a Jacques Derrida, Paul Ricoeur, JeanLouis Chrétien, Jean-François Courtine, y en general, a los últimos representantes de la fenomenología en Francia, suelo en el que, en todo caso, la fenomenología ha conocido un sin igual arraigo y que le ha tributado una larga tradición, asegurada desde las lecciones parisinas de Husserl en 1929 -las Meditaciones cartesianas-, y tan viva desde entonces en la actualidad ${ }^{3}$.

Las reacciones de Janicaud han sido provocadas por el hecho de que esta nouvelle phénoménologie admita como fenómeno la Revelación, postulado que Jean-Luc Marion justifica al introducir la noción de fenómeno saturado. Por

1 D. Janicaud, Le tournant théologique de la phénoménologie française, Paris, Éditions de L'Éclat, 1991.

2 D. Janicaud, La phénoménologie éclatée, Paris, Éditions de L'Éclat, 1998.

3 Para la historia de la fenomenología en Francia, véase entre muchos otros trabajos, V. Descombes, Lo mismo y lo otro. Cuarenta y cinco años de filosofía francesa (1933-1978), traducción de E. Benarroch, Madrid, Cátedra, 1982; Y. Mabin et al., Philosophie contemporaine en France, Paris, Ministère des Affaires Étrangères, 1994 y E. Alliez, De l'impossibilité de la phénoménologie. Sur la philosophie française contemporaine, Paris, Vrin, 1995 (versión aumentada). éste se entiende un tipo particular de fenómeno que sobrepasa la objetividad de lo dado y que concede su derecho al acontecimiento de la trascendencia, para hacer de lo revelado un modo privilegiado de la manifestación. La infinitud del rostro (Lévinas), pero también el don (Derrida), el icono (Marion), la carne (Henry), lo imposible y el llamado (Chrétien) corresponden a esta clase de fenómenos. Su intelección exige la apertura a una relación hasta ahora impracticada con el fenómeno, caracterizado ya no por su contenido de presencia, sino justamente por un exceso de donación. Saturados, los fenómenos del rostro, el don, el icono, la carne, etc., dan más de lo que salta simplemente a la presencia y de lo que asalta la mirada de quien los asiste en su aparecer. Aparecen como no visibles, y por tanto, invisibles, paradoja en la que justamente lo invisible inaugura un nuevo tipo de visibilidad. Por eso Jean-Luc Marion hablará en el mismo sentido de fenómenos paradójicos o saturados. Janicaud, en cambio, sólo reconocerá en ellos el abandono de los principios de la ciencia fenomenológica formulados por Husserl, y la seducción de sus coterráneos ante lo que Heidegger, en su Seminario de Zähringen (1973), esbozaba ya como una "fenomenología

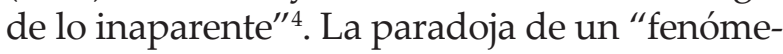
no inaparente" equivaldría, según Janicaud, a perder todo rigor para la fenomenología. Para él, lo que entre los franceses pasa hoy por fenomenología desconoce el ámbito de lo dado y desatiende las condiciones de la reducción, de suerte que, para él, este "retorno de la trascendencia" describirá tan sólo un movimiento retrógrado frente al proyecto de una filosofía trascendental, cuyos réditos terminan siendo entregados a una teología que inesperadamente es restituida a su antiguo lugar de "filosofía primera".

4 M. Heidegger, "Seminario de Zähringen (1973)", traducción de O. Lorca, A Parte Rei, 37, 2005, p. 17. 
Pero leído críticamente, aunque sin demasiado rigor, el título de Janicaud deja entrever varios elementos que lo hacen susceptible de una simplificación excesiva. El primero y el más cuestionable, es el de presuponer la impermeabilidad de la frontera entre filosofía y teología, como si ésta estuviese tan absolutamente demarcada, adhiriendo con ello a una posición prejuiciosa y fácil en una discusión inagotable cuyo examen requeriría una reconstrucción retrospectiva de por lo menos dos siglos de discusión; no insistiremos en ello. En segundo lugar, tan cuestionable como el anterior, es el hecho de pretextar la polémica bajo la grandilocuente palabra "giro" (tournant), que la filosofía del siglo XX ha vuelto casi un estribillo puesto a relucir cada vez que el orden del discurso sufre alguna transformación, a riesgo de diluir el acontecimiento filosófico genuino en lo que termina siendo apenas una de tantas modas intelectuales (el "giro ontológico", "hermenéutico", "lingüístico", "intersubjetivo"), tan frecuentes como pasajeras en la filosofía. Por último, se le criticará por reincidir en el odioso cliché de las filosofías "nacionales", enmarcando de antemano el "giro" en las coordenadas de una doble marca identitaria (la "fenomenologíafrancesa"), en lo que termina por reactualizar - aún sin quererlo- la ilusión siempre desmentida de una "patria para el pensamiento" (Grecia o Alemania), contraria a las aspiraciones de una política y de un pensamiento que se reputan hoy de cosmopolitas.

Afortunadamente, la recepción internacional del debate ha sabido superar todos estos clichés, otorgándole la resonancia profunda de una inflexión decisiva en el curso histórico de la filosofía. Particularmente notable es su traslado al ámbito filosófico norteamericano, más sensible desde la publicación neoyorkina de Phenomenology and the "Theological turn", volumen que compendia las versiones al inglés del texto inaugural de Dominique Janicaud y las del importante compilatorio francés Phénoménologie

5 D. Janicaud et al., Phenomenology and "Theological Turn". The French Debate, traducción inglesa de B. G. Prusak, New York, Fordham University Press, 2000. et théologie, dirigido en 1992 por Jean-François Courtine $^{6}$, y al que aportaron sus polémicos escritos los principales imputados (Marion, Chrétien, Ricoeur, Henry y el mismo Courtine). De otro lado, aunque en el ámbito norteamericano se encuentran hoy diversos estudios - algunos generales, otros parciales - sobre la discusión ${ }^{7}$, la marca dominante la ha ido dejando el pensamiento de Jean-Luc Marion, cuyas obras vienen siendo regularmente traducidas al inglés, y a quien le han sido consagrados importantes trabajos como los de John D. Caputo (junto con Michael Scanlon) y Robyn Horner, a propósito del diferendo con Jacques Derrida sobre la cuestión del don, o como los de Shane Mackinlay, Kevin Hart y Christina Gschwandtner, más enfocados a la penetración detallada en su pensamiento ${ }^{8}$. En el mismo rango de importancia queremos situar el aporte de la compilación francocanadiense La métaphysique, son histoire, sa critique et ses enjeux, a cargo de Jean-Marc Narbonne y Jean-Luc Langlais ${ }^{9}$, que convoca junto a JeanLuc Marion al mismo Dominique Janicaud, acompañados de Jean-François Courtine, Alain de Libera, Jean-Louis Vieillard-Baron, Jacques Taminiaux, Jean-François Mattéi, entre muchos otros, en un volumen imprescindible para el tratamiento contemporáneo del litigio entre filosofía y teología que pasa necesariamente por recurrentes cruces e intercambios en la historia de la metafísica.

Por su parte, en el ámbito hispano, aunque no se cuenta todavía con una versión castellana del texto de Janicaud que "oficializa" la

6 J.-F. Courtine (ed.), Phénoménologie et théologie, Paris, Criterion, 1992.

7 B. E. Benson \& N. Wirzba (Eds). Words of Life. New theological turns in French phenomenology, New York, Fordham University Press, 2010. También el "dossier" coordinado por J. Reynolds (Ed.) "The implicit and presupposed theological turn in phenomenology", Sophia: An International Journal for Philosophy of Religion, Metaphysical Theology and Ethics, 47 (3), Springer, 2008.

8 J. Caputo \& M. Scanlon (eds.), God, the Gift, and Postmodernism, Bloomington, Indiana University Press, 1999. R. Horner, Rethinking God as Gift. Marion, Derrida and the Limits of Phenomenology, New York, Fordham University Press, 2001. S. Mackinlay, Interpreting Excess: JeanLuc Marion, Saturated Phenomena and Hermeneutics, New York, Fordham University Press, 2010. K. Hart (ed.), Counter-Experiences. Reading Jean-Luc Marion, Notre Dame (Indiana), University of Notre Dame Press, 2007. C. M. Gschwandtner, Reading Jean-Luc Marion. Exceeding Metaphysics, Bloomington, Indiana University Press, 2007.

9 J.-M. Narbonne \& J.-L. Langlais (eds.), La métaphysique, son histoire, sa critique et ses enjeux, Paris/Québec: Vrin/Presses Universitaires de Laval, 1999. 
efectuación del "giro", España viene aportando desde hace años la materia prima para su estudio al ofrecer las traducciones de los autores que Janicaud ha puesto en el centro de la discusión. Invaluable el aporte de la colección "Hermeneia" de Ediciones Sígueme, dirigida por Miguel García-Baró, a través de la cual nos son accesibles las obras fundamentales de Emmanuel Lévinas, Michel Henry, y en menor número, las de Jean-Louis Chrétien y Jean-Luc Marion, y que se podría decir "complementada" por la colección "Esprit" de Caparrós Editores, que incluye éstos y muchos otros de los autores implicados así sea tangencialmente en el debate ${ }^{10}$. En el caso específico de Jean-Luc Marion, ningún esfuerzo mayor que el de las cuidadosas versiones castellanas de Javier Bassas Vila y Joana Masó en las editoriales Ellago y Síntesis ${ }^{11}$, al que se suma la todavía reciente versión castellana de La ontología gris, a cargo de Alejandro García Mayo, quien con este libro ha dado el primer paso para poner finalmente a disposición de los lectores en lengua castellana la famosa "trilogía" de Marion dedicada a Descartes $^{12}$. En cuanto a los estudios españoles

10 Para limitar nuestro inventario a las dos colecciones mencionadas, cfr. E. Lévinas, Totalidad e infinito. Ensayo sobre la exterioridad, traducción de D. E. Guillot, Salamanca, Sígueme, 1977; De otro modo que ser o más allá de la esencia, traducción de A. Pintor Ramos, Salamanca, Sígueme, 1987; La teoría fenomenológica de la intuición, traducción de T. Checchi, Salamanca, Sígueme, 2004; Los imprevistos de la historia, traducción de T. Checchi, Salamanca, Sígueme, 2006; Humanismo del otro hombre, traducción de G. González R. Arnáiz, Madrid, Caparrós, 1993; De Dios que viene a la idea, traducción de G. González R. Arnáiz y J. M. Ayuso Díez, Madrid, Caparrós, 1995; Fuera del sujeto, traducción de R. Ranz y C. Jarillot Rodal, Madrid, Caparrós, 1997; Difícil libertad, traducción de J. Haidar, Madrid, Caparrós, 2004. M. Henry, Encarnación. Una filosofía de la carne, traducción de J. Teira, G. Fernández y R. Ranz, Salamanca, Sígueme, 2001; Yo soy la verdad, traducción de J. Teira, Salamanca, Sígueme, 2001; Palabras de Cristo, traducción de R. Ranz y J. Teira, Salamanca, Sígueme, 2004; Filosofía y fenomenología del cuerpo, traducción de J. Gayo Reyzábal, Salamanca, Sígueme, 2007; La barbarie, traducción de T. Domingo Moratalla, Madrid, Caparrós, 1996. J.-L. Chrétien, Lo inolvidable y lo inesperado, traducción de J. Teira y R. Ranz, Salamanca, Sígueme, 2002; La mirada del amor, traducción de P. Jimeno Barrera, Salamanca, Sígueme, 2005; La llamada y la respuesta, traducción de J. A. Sucasas, Madrid, Caparrós, 1997. J.-L. Marion, El ídolo y la distancia, traducción de S. M. Pascual y N. Latrille, Salamanca, Sígueme, 1999; Prolegómenos a la caridad, traducción de C. Díaz, Madrid, Caparrós, 1993.

11 Cfr. J.-L. Marion, El cruce de lo visible, traducción de J. Bassas Vila y J. Masó Illamola, Castellón, Ellago, 2006; Siendo dado. Ensayo para una fenomenología de la donación, prólogo a la edición española de J.-L. Marion, traducción, presentación y notas de J. Bassas Vila, Madrid, Síntesis, 2008; "El icono o la hermenéutica sin fin", Alea, Revista internacional de fenomenología y hermenéutica, 5, 2007, pp. 137-166. Y con apoyo nuestro, Dios sin el ser, traducción de D. Barreto, J. Bassas Vila y C. E. Restrepo, revisión y postfacio de J. Bassas Vila, Vilaboa, Ellago, 2010.

12 Cfr. J.-L. Marion, Sobre la ontología gris de Descartes, traducción de A. García Mayo, Madrid, Escolar y Mayo, 2008. En comentario, véase nuestra reseña en La torre del virrey (Separata de libros), 2ª Serie, Pri- de la obra de Marion, los más notables son, sin duda, los de Daniel Barreto, del Instituto Superior de Teología de Islas Canarias, quien desde hace varios años viene siguiendo con especial atención el debate de Marion con Derrida ${ }^{13}$. Entre tanto, la obra de Emmanuel Lévinas, sobre la que otrora fueron publicados los estudios de José María Aguilar López y Ulpiano Vázquez Moro - este último, autor de un libro considerado clásico-, no podría quedar actualmente mejor recogida que en los trabajos de Alberto Sucasas (traductor además de J.-L. Chrétien en Caparrós Editores), Patricio Peñalver (quien tanto ha hecho además por la divulgación de la obra de Derrida), y Julia Urabayen (siendo notable en este último caso su coexistencia con el reclamo de un nuevo auge para la obra de Gabriel Marcel) ${ }^{14}$. Se recordará también la revisión de Marion, Henry y Chrétien que realizara en su momento Pilar Fernández Beites, en la que es muy posiblemente la primera lectura del "giro" en España, publicada en el monográfico de la Revista de Occidente sobre el litigio contemporáneo entre filosofía y teología ${ }^{15}$.

En América Latina, aunque las investigaciones existentes resultan demasiado dispersas e insulares, algunos trabajos importantes se han ocupado de los protagonistas del "giro" o de otros autores que han ido tomando parte en

mavera de 2009 (disponible en Internet: http://www.estudiosculturales.es/libros/serie2.php)

13 D. Barreto, "Entre Jean-Luc Marion y Jacques Derrida. A propósito de la alabanza y la oración como actos de habla", Philologica canariensia 10/11, 2004-2005, pp. 533-546; "La donación y lo imposible. Aproximación a la filosofía de Jean-Luc Marion", Almogaren, 37, 2005, pp. 11-37; "El debate entre Jean-Luc Marion y Jacques Derrida. Una introducción", Revista Laguna, 18, 2006, pp. 35-47.

14 Respectivamente, J. M. Aguilar López, Trascendencia y alteridad: estudio sobre E. Lévinas, Pamplona, Eunsa, 1992. U. Vázquez Moro, El discurso sobre Dios en la obra de E. Lévinas, Madrid, Universidad Pontificia de Comillas, 1982. A. Sucasas, Lévinas. Lectura de un palimpsesto, Buenos Aires, Lilmod, 2006. P. Peñalver, Argumento de alteridad. La hipérbole metafísica de Emmanuel Lévinas, Madrid, Caparrós, 2001. J. Urabayen, Las raíces del humanismo de Emmanuel Levinas: el judaísmo y la fenomenología, Pamplona, Eunsa, 2005; El pensamiento antropológico de Gabriel Marcel: un canto al ser humano, Pamplona, Eunsa, 2001; Las sendas del pensamiento hacia el misterio del ser. La filosofía concreta de Gabriel Marcel, Pamplona, Cuadernos de Anuario Filosófico, 2001. En materia de compilaciones, con textos de A. Sucasas, P. Peñalver y J. M. Aguilar López, dos volúmenes nos parecen importantes: D. Pérez Chico \& M. Barroso Ramos (eds.), Un libro de huellas: aproximación al pensamiento de Emmanuel Lévinas, Madrid, Trotta, 2004; y el monográfico "Emmanuel Lévinas: Un compromiso con la otredad", Revista Anthropos, 176, Barcelona, 1998.

15 P. Fernández Beites, "Fenomenología francesa y teología", Revista de occidente, 258, 2002, pp. 124-147. 
él en la escena europea. De los escritos que Paul Gilbert ha dedicado a la cuestión, una parte importante ha sido recogida en el volumen $\mathrm{Algu-}$ nos pensadores contemporáneos de lengua francesa, publicado por la Universidad Iberoamericana de México y editado por José Rubén Sanabria ${ }^{16}$, quien publicara en la misma colección una Historia de la filosofía cristiana en México junto con Mauricio Beuchot; otros del mismo autor han aparecido en Colombia y Argentina, en ediciones del Equipo Jesuita Latinoamericano de Reflexión Filosófica ${ }^{17}$. Por su parte, importantes contribuciones de Phillipe Capelle, Jean-Luc Marion y Jean Greisch han sido traducidas y publicadas en Buenos Aires por la Universidad Nacional de San Martín bajo el sello Jorge Baudino Ediciones, a las que se añade la versión también argentina del libro de Marion El fenómeno erótico ${ }^{18}$. Y es que en América Latina, Argentina es ciertamente el país que mejor ha sabido actualizar el debate. En ello han sido decisivos los incontables trabajos de Oscar del Barco, Juan Carlos Scannone, Roberto Walton, Raúl Ballbé, Mario Lipsitz, Ricardo Díez, Pablo Dreizik, Ángel Garrido-Maturano, Mariana Leconte, Julia Iribarne, Marie-France Begué, Francisco Díez-Fischer, Anibal Fornari, entre otros, en los que resulta predominante -en su orden - la recepción de Marion, Henry, Lévinas y Ricoeur ${ }^{19}$; sin olvidar como telón de fondo

16 P. Gilbert, Algunos pensadores contemporáneos de lengua francesa, traducción de J. Rubén Sanabria, México, Universidad Iberoamericana, 1996. Para una reconstrucción más reciente, cfr. P. Gilbert, "Actualité d'une philosophie chrétienne", Raisons politiques, 4, 2001, pp. 15-39. Aunque en otro sentido, nos parece importante no pasar por alto su libro $\mathrm{Me}$ tafísica. La paciencia de ser, traducción de E. Peña Eguren, Salamanca, Sígueme, 2008.

17 V. Durán et al., Problemas de la filosofía de la religión desde América Latina, Bogotá, Siglo del Hombre, 2003.

18 Ph. Capelle, Fenomenología francesa actual, Buenos Aires, Jorge Baudino Ediciones/UNSAM, 2009. J.-L. Marion, Acerca de la donación, Buenos Aires, Jorge Baudino Ediciones/UNSAM, 2005. J. Greisch, El cogito herido, Buenos Aires, Jorge Baudino Ediciones, 2001. J.-L. Marion, El fenómeno erótico, traducción de S. Mattoni, Buenos Aires, El cuenco de la plata, 2005.

19 Ante la imposibilidad de un inventario, citamos sólo algunos ejemplos: O. del Barco, Exceso y donación, Buenos Aires, Biblioteca Internacional Martin Heidegger, 2003. J. C. Scannone, “Los fenómenos saturados de Jean-Luc Marion y la fenomenología de la religión", Stromata, 61 (1/2), 2005, pp. 1-15; también "Fenomenología y hermenéutica en la fenomenología de la donación de Jean-Luc Marion", Stromata, 61 (1/2), 2005, pp. 179-193. R. Walton, "Apertura, desvelamiento y responsabilidad", en P. Bonzi y J. J. Fuentes (eds.), El énfasis del infinito. Esbozos y perspectivas en torno al pensamiento de Emmanuel Lévinas, Barcelona, Anthropos, 2009, pp. 51-75; también "Reducción fenomenológica y figuras de la excedencia", Tópicos (Santa Fe), 16, 2008, 169-187. R. Ballbé et al., Michel Henry, Lausanne, L'âge d'homme, 2009. M. Lipsitz, Eros y expedientes de traducciones e inéditos como los que han ido dejando la revista Nombres de la Facultad de Filosofía y Humanidades de la Universidad Nacional de Córdoba, y la Biblioteca Internacional Martin Heidegger que ha divulgado los textos del "último Heidegger" en las versiones de Dina Picotti ${ }^{20}$.

En lo concerniente a los proyectos que cruzan "continentalmente" a América Latina, los autores del "giro" van siendo poco a poco considerados (aunque no siempre aceptados) por los fenomenólogos que congrega el Círculo Latinoamericano de Fenomenología (CLAFEN), del que hacen parte especialistas de Perú, Bolivia, Ecuador, Colombia, Venezuela, Brasil, Chile, Argentina, República Dominicana, Guatemala, México, Costa Rica, Norteamérica y algunos países de Europa. Esta presencia paulatina y discreta se atestigua desde la aparición del órgano de divulgación del Círculo, el Acta fenomenológica latinoamericana, que ya en su primer volumen incluyó un escrito de Michel Henry, y que ha publicado también comentarios sobre éste y otros fenomenólogos franceses en sus siguientes ediciones ${ }^{21}$. También hay que

nacimiento fuera de la ontología griega: Emmanuel Lévinas y Michel Henry, Buenos Aires, Prometeo, 2004; también "Michel Henry y la crítica del intuicionismo", A parte rei, 10, 2000. R. Díez, "Michel Henry, fundador de la fenomenología de la Vida", Acta fenomenológica latinoamericana, 3, 2009, pp. 233-245. P. Dreizik, "Levinas y el problema de la violencia trascendental en la determinación fenoménica de la alteridad", Jornadas Emmanuel Levinas: Ética, metafísica y judaísmo, Buenos Aires, 2005. A. Garrido-Maturano, "El eros como trascendencia no ética y tránsito fecundo. Lectura de la fenomenología del eros de E. Lévinas", Thémata, 18, 1997, pp. 75-90. M. Leconte, Racionalidad y proximidad. La significancia ética como origen de la significación en Emmanuel Lévinas, Corrientes, Moglia Ediciones, 2009. J. Iribarne, “La cuestión de Dios y la fenomenología contemporánea", en M. Giusti (ed.), La filosofía del siglo XX: balance y perspectivas, Lima, Universidad Católica del Perú, 2000, pp. 324-330. M.-F. Begué, Paul Ricoeur. La poética del sí-mismo, Buenos Aires, Biblos, 2002. F. Díez-Fischer, "El hogar en el mundo de la vida", Anuario colombiano de fenomenología, 3, 2009, pp. 85-103. A. Fornari y R. Walton, Volver hoy a las cosas mismas. Verdad, libertad, educación. Buenos Aires, Biblos, 2009.

20 Para las traducciones de Dina Picotti, cfr. M. Heidegger, Aportes a la filosofía. Acerca del evento, Buenos Aires, Biblioteca Internacional Martin Heidegger, 2003; y en la misma colección, Meditación (2006) y Sobre el comienzo (2007). Por su parte, desde 1991, la Revista Nombres ha ido publicando traducciones de Heidegger, Marion, Lévinas, J.-L. Nancy, Deleuze, Agamben, Foucault, etc., con importantes comentarios de Oscar del Barco, Diego Tatián y muchos otros intérpretes. El índex completo de la Revista Nombres se puede consultar en el link: http:// www.ffyh.unc.edu.ar/dependencias/ciffyh/Nueva/Publicaciones_todas/Nombres.htm

21 M. Henry, "Por una fenomenología de la vida", Acta fenomenológica latinoamericana, 1, Lima, Pontificia Universidad Católica del Perú, 2003, pp. 429-436. Para las restantes ediciones del Acta y las demás actividades de CLAFEN, véase el link: www.clafen.org. 
destacar importantes encuentros como el "IV Simposio Internacional de Estudios Cruzados sobre la Modernidad" dedicado a Jean-Luc Marion, coordinado por Juan Carlos Moreno Romo (reconocido traductor de Jean-Luc Nancy), y realizado en la Universidad Autónoma de Querétaro (México) entre los días 15 y 19 de noviembre de 2010. De otro lado, aunque es todavía sensible el desconocimiento (recíproco) entre los países latinoamericanos y Brasil, podemos sumar a nuestro inventario el volumen "paulistano" Teologia e pós-modernidade, a cargo de Jaci Maraschin y Frederico Pieper Pires, que incluye textos de Robyn Horner y John D. Caputo ${ }^{22}$, así como el trabajo sobre Lévinas concentrado en el colectivo CEBEL (Centro Brasilero de Estudios sobre el Pensamiento de Emmanuel Lévinas), a cargo de Marcelo Fabri, Ricardo Timm de Souza, Pergentino Pivatto y Luiz Carlos Susin, en un centro asociado a la Pontificia Universidad Católica de Porto Alegre (Rio Grande do Sul) ${ }^{23}$.

En Colombia, aunque proscritas por el "dogmatismo paleomoderno" de las facultades de filosofía - según la expresión de Anibal Fornari- y refundidas en unas cuantas publicaciones universitarias, cuando no invisibilizadas por el poco o nulo interés que tiene por la filosofía la exigua "industria editorial" del país, ha habido a pesar de todo importantes contribuciones. Las más notables, las dirigidas por Carlos Arboleda Mora en publicaciones recientes de la Universidad Pontificia Bolivariana (Medellín), entre las que se cuentan su tesis doctoral Profundidad y cultura (2007), y las compilaciones Pragmatismo, posmetafísica y religión (2008), Posontología y posmetafísica (2009), así como diversos artículos suyos en la revista Escritos de la Facultad de Filosofía de dicha universidad ${ }^{24}$.

22 J. Maraschin \& F. Pieper Pires (eds.), Teologia e pós-modernidade. Novas perspectivas em teologia e filosofia da religião, São Paulo, Fonte Editorial, 2008.

23 Sólo como ilustración citaremos L.C. Susin et al., Éticas em diálogo. Levinas e o pensamento contemporâneo: questões e interfaces, Porto Alegre, EDIPUCRS, 2003, que incluye también un texto del argentino P. Dreizik, "Freud y Levinas. Una exigencia (Anspruch) ética que corta el hilo de la conciencia", pp. 97-110. Es del caso recordar la participación del mentado colectivo en el II Simpósio Internacional de Fenomenologia e Hermenêutica, realizado entre octubre y noviembre de 2001 en la Universidad Federal de Goiás (Goiânia), que contó también con la participación de Dominique Janicaud y John D. Caputo.
Otro espacio importante para la discusión lo ha brindado el Anuario colombiano de fenomenología, fundado en 2007 por Pedro Juan Aristizábal (Universidad Tecnológica de Pereira) y sostenido desde entonces por un colectivo animado por Germán Vargas Guillén y Luz Gloria Cárdenas (Universidad Pedagógica Nacional y Universidad de Antioquia, respectivamente), el cual en su tercer volumen contiene un nutrido "dossier" sobre Jean-Luc Marion, al que de nuestra parte hemos aportado algún artículo y la traducción de la famosa discusión con Derrida "Sobre el don", presentada por Daniel Barreto $^{25}$. Encomiable nos parece también el trabajo conjunto de Edgar Antonio López (Universidad Javeriana, Facultad de Teología) y Mateo Navia Hoyos (Universidad de Antioquia), quienes desde 2005 y sin interrupción promueven el escenario anual de discusión denominado Jornadas Nacionales Emmanuel Lévinas, que lamentablemente no ha trasladado su producción a medios impresos. En otra prespectiva, inaugurada en nuestro país por la interpretación de Mario Madroñero Morillo (Universidad de Nariño, Pasto), los autores del "giro" vienen siendo explorados en función de una "fenomenología de la donación artística", o si se quiere, de una "fenomenología del arte"26. Esta veta, de por sí fascinante, ha sido provocada por la incidencia de las reflexiones de Lévinas y Marion en el campo del arte, derivada del uso de las nociones de ídolo e icono, pero también por trabajos como el de Michel Henry sobre Kandinsky, o como los de Jean-Luc Nancy (a quien no hay por qué involucrar en el "giro") en su reflexión contem-

24 C. Arboleda Mora, Profundidad y cultura. Del concepto de Dios a la experiencia de Dios, Medellín, Universidad Pontificia Bolivariana, 2007; Pragmatismo, posmetafísica y religión, Medellín, Universidad Pontificia Bolivariana, 2008; Posontología y posmetafísica en el siglo XXI, Medellín, Universidad Pontificia Bolivariana, 2009; “Dios: ¿Ser o don?”, Escritos, 17 (38), 2009, pp. 14-53.

25 Cfr. Anuario colombiano de fenomenología, 3, 2009, pp. 243-274. A la discusión Marion-Derrida, el "dossier" en cuestión integra textos de Harry Reeder, Germán Vargas Guillén, Carlos Enrique Restrepo, Javier Bassas Vila, Daniel Barreto y Mario Madroñero. En su último número, coordinado esta vez por Guillermo Pérez La Rotta y Juan Carlos Aguirre (Universidad del Cauca), el Anuario incluye el artículo de J.-L. Marion, "El fin del fin de la metafísica", Anuario colombiano de fenomenología, 4, 2010, pp. 367-380.

26 M. Madroñero, "Estética de la invisibilidad. Notas sobre la creación artística a partir de El cruce de lo visible de Jean-Luc Marion", Anuario colombiano de fenomenología, 3, 2009, pp. 339-355. 
poránea acerca de la imagen ${ }^{27}$. También con un interés estético, recientemente empezamos a conocer los trabajos de María Cristina Sánchez León (Universidad de La Salle, Bogotá), quien en múltiples frentes adelanta investigaciones muy contemporáneas a la luz de autores como Ricoeur, Merleau-Ponty, Michel Serres y JeanLuc Marion ${ }^{28}$.

Como se ve, pues, las aristas del problema son muchas y el campo de investigación fecundo. Ciertamente, todavía hay que echar de menos la traducción castellana de muchísimos textos de los fenomenólogos franceses del "giro", empezando por los del mismo Janicaud, pero se ha avanzado quizás más de lo esperado, a pesar del carácter parcial de las investigaciones y de la restringida circulación que las publicaciones en esta materia tienen en América Latina. Con este acervo, están dadas las condiciones para trasladar la discusión a los escenarios de enseñanza filosófica universitaria, aspecto en el que - con excepción de Argentina- el impacto es poco sensible aún. Para ello, el reencuentro con la teología (cristiana y judía) y con fuentes de pensamiento como la filosofía medieval es no sólo perentorio, sino también muy prometedor. En este sentido, son de destacar iniciativas como las del Centro de Estudios Filosóficos Eugenio Pucciarelli de la Academia Nacional de Ciencias de Buenos Aires (Argentina), y en especial, de la Sección de Filosofía Medieval que dirige Ricardo Díez, la cual ha hecho importantes contribuciones en este campo.

Por lo visto, ha pasado el tiempo de los prejuicios heideggerianos según los cuales la teología es el "enemigo mortal" (Todfeind) de

27 J.-L. Marion, El cruce de lo visible, ob. cit. E. Lévinas, La realidad y su sombra, introducción de A. Domínguez Rey, traducción de A. Domínguez Leiva, Madrid, Trotta, 2001. M. Henry, Ver lo invisible. Acerca de Kandinsky, traducción de M. Tabuyo y A. López, Madrid, Siruela, 2008. J.-L. Nancy, La mirada del retrato, traducción de I. Agoff, Buenos Aires, Amorrortu, 2006; Noli me tangere, traducción de M. Tabuyo, A. López Tobajas y A. del Río Herrmann, Madrid, Trotta, 2006; “La imagen - Lo distinto", Revista Laguna, 11, 2002, pp. 9-22.

28 M. C. Sánchez León, "Paul Ricoeur: De L’herméneutique du témoignage à l'ontologie de l'attestation", en Y. B. Raynova (ed.), Paul Ricoeur: Interpretation, Werte Handeln, Wien, Peter Lang, 2004; también "Rostro y testimonio: mística y poética del don en la fenomenología de JeanLuc Marion", Jean-Luc Marion: El don de una tradición y de un futuro para el pensamiento, Querétaro (México), 2010. la filosofía y decir "filosofía cristiana" es como "hierro de madera" ${ }^{29}$. En esto, como en muchas otras cuestiones fundamentales, Heidegger se equivoca de raíz, inducido por su anacrónica y abyecta devoción al pensamiento griego. Contra semejantes prejuicios, hay que recordar que la filosofía occidental hereda también otras fuentes, y de entre éstas, la más profunda es la judeocristiana. El "giro teológico" evoca el cruce de tales fuentes, reviviendo al hacerlo posiciones encontradas y un antiguo caso de litigio. No insistiremos en lo litigioso sino en el cruce, aunque a algunos la alteridad, la hibridación y la mezcla les resulten insoportables. Tan abyecta como la postura heideggeriana nos parece, por tanto, la de Janicaud en su defensa sin tregua del $c a-$ non husserliano de la fenomenología. Muy a su pesar, el hecho es que no hay filosofía pura, ni fenomenología pura, sino "pensamiento", y que éste no conoce ni canon, ni dogmas, ni patria, sino que precisamente se autoafirma a condición de su libertad.

\section{Bibliografia}

Aguilar López, J. M. Trascendencia y alteridad. Estudio sobre E. Lévinas, Pamplona, Eunsa, 1992.

Alliez, E. De l'impossibilité de la phénoménologie. Sur la philosophie française contemporaine, Paris, Vrin, 1995.

Arboleda Mora, C. Profundidad y cultura. Del concepto de Dios a la experiencia de Dios, Medellín, Universidad Pontificia Bolivariana, 2007.

Arboleda Mora, C. Pragmatismo, posmetafisica y religión, Medellín, Universidad Pontificia Bolivariana, 2008.

Arboleda Mora, C. Posontología y posmetafisica en el siglo XXI, Medellín, Universidad Pontificia Bolivariana, 2009.

Arboleda Mora, C. “Dios: ¿Ser o don?”, Escritos, 17 (38), 2009, pp. 14-53.

29 M. Heidegger, "Fenomenología y teología", Hitos, Madrid, Alianza, 2001, p. 65. 
Ballbé, R. et al., Michel Henry, Lausanne: L'âge d'homme, 2009.

Barreto, D. “Entre Jean-Luc Marion y Jacques Derrida. A propósito de la alabanza y la oración como actos de habla", Philologica canariensia, 10/11, 2004-2005, pp. 533-546.

Barreto, D. "La donación y lo imposible. Aproximación a la filosofía de Jean-Luc Marion", Almogaren, 37, 2005, pp. 11-37.

Barreto, D. “El debate entre Jean-Luc Marion y Jacques Derrida. Una introducción", Revista Laguna, 18, 2006, pp. 35-47.

Bassas Vila, J. “El origen original de la imagen. Lo virtual en fenomenología”, Anuario colombiano de fenomenología, 3, 2009, pp. 329-338.

Begué, M.-F. Paul Ricoeur. La poética del símismo, Buenos Aires, Biblos, 2002.

Benson, B. E. \& Wirzba, N. (eds). Words of Life. New Theological Turns in French Phenomeno$\log y$, New York, Fordham University Press, 2010.

Capelle, Ph. Fenomenología francesa actual, Buenos Aires, Jorge Baudino Ediciones/UNSAM, 2009.

Caputo, J. \& Scanlon, M. (eds.). God, the Gift, and Postmodernism, Bloomington, Indiana University Press, 1999.

Cárdenas Mejía, L. G. "Hacia una hermenéutica del lugar", Anuario colombiano de fenomenología, 4, 2010, pp. 133-147.

Chrétien, J.-L., La llamada y la respuesta, traducción de J. A. Sucasas, Madrid, Caparrós, 1997.

Chrétien, J.-L., Lo inolvidable y lo inesperado, traducción de J. Teira y R. Ranz, Salamanca, Sígueme, 2002.

Chrétien, J.-L., La mirada del amor, traducción de P. Jimeno Barrera, Salamanca, Sígueme, 2005.
Courtine, J.-F. (Ed.), Phénoménologie et théologie, Paris, Criterion, 1992.

Del Barco, O. Exceso y donación, Buenos Aires, Biblioteca Internacional Martin Heidegger, 2003.

Descombes, V., Lo mismo y lo otro. Cuarenta y cinco años de filosofía francesa (1933-1978), traducción de E. Benarroch, Madrid, Cátedra, 1982.

Díez, R. "Michel Henry, fundador de la fenomenología de la Vida", Acta fenomenológica latinoamericana, 3, 2009, pp. 233-245.

Díez-Fischer, F. “El hogar en el mundo de la vida", Anuario colombiano de fenomenología, 3, 2009, pp. 85-103.

Dreizik, P., "Freud y Lévinas. Una exigencia (Anspruch) ética que corta el hilo de la conciencia", en L. C. Susin y otros (eds.), Éticas em diálogo. Lévinas e o pensamento contemporâneo: questões e interfaces, Porto Alegre, EDIPUCRS, 2003, pp. 97-110.

Dreizik, P. "Levinas y el problema de la violencia trascendental en la determinación fenoménica de la alteridad", Jornadas Emmanuel Levinas: Ética, metafísica y judaísmo, Buenos Aires, 2005.

Durán, V. et al., Problemas de la filosofía de la religión desde América Latina, Bogotá, Siglo del Hombre, 2003.

Fernández Beites, P. “Fenomenología francesa y teología”, Revista de Occidente, 258, 2002, pp. 124-147.

Fornari, A. \& Walton, R. Volver hoy a las cosas mismas. Verdad, libertad, educación. Buenos Aires, Biblos, 2009.

Garrido-Maturano, A. "El eros como trascendencia no ética y tránsito fecundo. Lectura de la fenomenología del eros de E. Lévinas", Thémata, 18, 1997, pp. 75-90. 
Gilbert, P. Algunos pensadores contemporáneos de lengua francesa, traducción de J. Rubén Sanabria, México, Universidad Iberoamericana, 1996.

Gilbert, P. "Actualité d'une philosophie chrétienne", Raisons politiques, 4, 2001, pp. 15-39.

Gilbert, P., Metafísica. La paciencia de ser, traducción de E. Peña Eguren, Salamanca, Sígueme, 2008.

Greisch, J. El cogito herido, Buenos Aires, Jorge Baudino Ediciones, 2001.

Gschwandtner, C. M. Reading Jean-Luc Marion. Exceeding Metaphysics, Bloomington, Indiana University Press, 2007.

Hart, K. (ed.). Counter-Experiences. Reading Jean-Luc Marion, Notre Dame (Indiana), University of Notre Dame Press, 2007.

Heidegger, M., "Fenomenología y teología", Hitos, traducción de H. Cortés y A. Leyte, Madrid, Alianza, 2001.

Heidegger, M., Aportes a la filosofía. Acerca del evento, traducción de D. V. Picotti, Buenos Aires, Biblioteca Internacional Martin Heidegger, 2003.

Heidegger, M., "Seminario de Zähringen (1973)", traducción de O. Lorca, A Parte Rei, 37, 2005.

Heidegger, M., Meditación, traducción de D. V. Picotti, Buenos Aires, Biblioteca Internacional Martin Heidegger, 2006.

Heidegger, M., Sobre el comienzo, traducción de D. V. Picotti, Buenos Aires, Biblioteca Internacional Martin Heidegger, 2007.

Henry, M., La barbarie, traducción de T. Domingo Moratalla, Madrid, Caparrós, 1996.

Henry, M., Encarnación. Una filosofía de la carne, traducción de J. Teira, G. Fernández y R. Ranz, Salamanca, Sígueme, 2001.
Henry, M., Yo soy la verdad, traducción de J. Teira, Salamanca, Sígueme, 2001.

Henry, M., "Por una fenomenología de la vida", Acta fenomenológica latinoamericana, 1, 2003, pp. 429-436.

Henry, M., Palabras de Cristo, traducción de R. Ranz y J. Teira, Salamanca, Sígueme, 2004.

Henry, M., Filosofía y fenomenología del cuerpo, traducción de J. Gayo Reyzábal, Salamanca, Sígueme, 2007.

Henry, M., Ver lo invisible. Acerca de Kandinsky, traducción de M. Tabuyo y A. López, Madrid, Siruela, 2008.

Horner, R. Rethinking God as Gift. Marion, Derrida and the Limits of Phenomenology, New York, Fordham University Press, 2001.

Iribarne, J., "La cuestión de Dios y la fenomenología contemporánea", en M. Giusti (ed.), La filosofía del siglo XX: balance y perspectivas, Lima, Universidad Católica del Perú, 2000, pp. 324-330.

Janicaud, D. Le tournant théologique de la phénoménologie française, Paris, Éditions de L'Éclat, 1991.

Janicaud, D. La phénoménologie éclatée, Paris, Éditions de L'Éclat, 1998.

Janicaud, D. et al., Phenomenology and "Theological Turn". The French Debate, traducción inglesa de B. G. Prusak, New York, Fordham University Press, 2000.

Leconte, M. Racionalidad y proximidad. La significancia ética como origen de la significación en Emmanuel Lévinas, Corrientes, Moglia Ediciones, 2009.

Lévinas, E., Totalidad e infinito. Ensayo sobre la exterioridad, traducción de D. E. Guillot, Salamanca, Sígueme, 1977. 
Lévinas, E., De otro modo que ser o más allá de la esencia, traducción de A. Pintor Ramos, Salamanca, Sígueme, 1987.

Lévinas, E., Humanismo del otro hombre, traducción de G. González y R. Arnáiz, Madrid, Caparrós, 1993.

Lévinas, E., De Dios que viene a la idea, traducción de G. González, R. Arnáiz y J. M. Ayuso Díez, Madrid, Caparrós, 1995.

Lévinas, E., Fuera del sujeto, traducción de R. Ranz y C. Jarillot, Madrid, Caparrós, 1997.

Lévinas, E., La realidad y su sombra. Libertad y mandato. Transcendencia y altura, introducción de A. Domínguez Rey, traducción de A. Domínguez Leiva, Madrid, Trotta, 2001.

Lévinas, E., Difícil libertad, traducción de J. Haidar, Madrid, Caparrós, 2004.

Lévinas, E., La teoría fenomenológica de la intuición, traducción de T. Checchi, Salamanca, Sígueme, 2004.

Lévinas, E., Los imprevistos de la historia, traducción de T. Checchi, Salamanca, Sígueme, 2006.

Lipsitz, M. “Michel Henry y la crítica del intuicionismo", A parte rei, 10, 2000.

Lipsitz, M. Eros y nacimiento fuera de la ontología griega: Emmanuel Lévinas y Michel Henry, Buenos Aires, Prometeo, 2004.

Mabin, Y., et al., Philosophie contemporaine en France, Paris, Ministère des Affaires Étrangères, 1994.

Mackinlay, S. Interpreting Excess: Jean-Luc Marion, Saturated Phenomena and Hermeneutics, New York, Fordham University Press, 2010.

Madroñero, M. “Estética de la invisibilidad. Notas sobre la creación artística a partir de El cruce de lo visible de Jean-Luc Marion",
Anuario colombiano de fenomenología, 3, 2009, pp. 339-355.

Maraschin, J. \& Pieper Pires, F. (eds.). Teologia e pós-modernidade. Novas perspectivas em teologia e filosofia da religião, São Paulo, Fonte Editorial, 2008.

Marion, J.-L. y Derrida, J., "Sobre el don", Anuario colombiano de fenomenología, 3, 2009, pp. 243-274.

Marion, J.-L., Prolegómenos a la caridad, traducción de C. Díaz, Madrid, Caparrós, 1993.

Marion, J.-L., El ídolo y la distancia. Cinco estudios, traducción de S. M. Pascual y N. Latrille, Salamanca, Sígueme, 1999.

Marion, J.-L., Acerca de la donación, Buenos Aires, Jorge Baudino Ediciones/UNSAM, 2005.

Marion, J.-L., El fenómeno erótico. Seis meditaciones, traducción de S. Mattoni, Buenos Aires, El cuenco de la plata, 2005.

Marion, J.-L., El cruce de lo visible, traducción de J. Bassas Vila y J. Masó Illamola, Castellón, Ellago, 2006.

Marion, J.-L., “El icono o la hermenéutica sin fin", Alea. Revista internacional de fenomenología y hermenéutica, 5, 2007, pp. 137-166.

Marion, J.-L., Siendo dado. Ensayo para una fenomenología de la donación, prólogo a la edición española de J.-L. Marion, traducción, presentación y notas de J. Bassas Vila, Madrid, Síntesis, 2008.

Marion, J.-L., Sobre la ontología gris de Descartes, traducción de A. García Mayo, Madrid, Escolar y Mayo, 2008.

Marion, J.-L., "El fin del fin de la metafísica", Anuario colombiano de fenomenología, 4, 2010, pp. 367-380.

Marion, J.-L., Dios sin el ser, traducción de D. Barreto González, J. Bassas Vila y C. E. Res- 
trepo, revisión y postfacio de J. Bassas Vila, Vilaboa, Ellago, 2010.

Nancy, J.-L., "La imagen - Lo distinto", traducción y notas de M. Barroso, Revista Laguna, 11, 2002, pp. 9-22.

Nancy, J.-L., La mirada del retrato, traducción de I. Agoff, Buenos Aires, Amorrortu, 2006.

Nancy, J.-L., Noli me tangere. Ensayo sobre el levantamiento del cuerpo, traducción de M. Tabuyo, A. López Tobajas y A. del Río Herrmann, Madrid, Trotta, 2006.

Narbonne, J.-M. \&. Langlais, J.-L (eds.). La métaphysique, son histoire, sa critique et ses enjeux, Paris/Québec: Vrin/Presses Universitaires de Laval, 1999.

Peñalver, P. Argumento de alteridad. La hipérbole metafísica de Emmanuel Lévinas, Madrid, Caparrós, 2001.

Pérez Chico, D. \& Barroso Ramos, M. (eds.). Un libro de huellas: aproximación al pensamiento de Emmanuel Lévinas, Madrid, Trotta, 2004.

Reeder, H. "El llamado 'fenómeno' saturado", Anuario colombiano de fenomenología, 3, 2009, pp. 275-298.

Restrepo, C. E. "Visibilidad de lo invisible. Incursión a los fenómenos de revelación", Anuario colombiano de fenomenología, 3, 2009, pp. 299-310.

Reynolds, J. (ed.). "The implicit and presupposed theological turn in phenomenology", Sophia: An International Journal for Philosophy of Religion, Metaphysical Theology and Ethics, 47 (3), Springer, 2008.

Sánchez León, M. C. “Paul Ricoeur: De L'herméneutique du témoignage à l'ontologie de l'attestation", Paul Ricoeur: Interpretation, Werte Handeln (Yvanka B. Raynova, Ed.), Wien, Peter Lang, 2004.
Sánchez León, M. C. “Rostro y testimonio: mística y poética del don en la fenomenología de Jean-Luc Marion”, Jean-Luc Marion: El don de una tradición y de un futuro para el pensamiento, Querétaro (México), 2010.

Scannone, J.C. "Los fenómenos saturados de Jean-Luc Marion y la fenomenología de la religión", Stromata, 61 (1/2), 2005, pp. 1-15.

Scannone, J.C. "Fenomenología y hermenéutica en la 'fenomenología de la donación' de Jean-Luc Marion", Stromata, 61 (1/2), 2005, pp. 179-193.

Sucasas, A. et al., "Emmanuel Lévinas: Un compromiso con la otredad", Revista Anthropos, 176, Barcelona, 1998.

Sucasas, A. Lévinas. Lectura de un palimpsesto, Buenos Aires, Lilmod, 2006.

Susin, L.C. et al., Éticas em diálogo. Levinas e o pensamento contemporâneo: questões e interfaces, Porto Alegre, EDIPUCRS, 2003.

Urabayen, J. Las sendas del pensamiento hacia el misterio del ser. La filosofía concreta de Gabriel Marcel, Pamplona, Cuadernos de Anuario Filosófico, 2001.

Urabayen, J. El pensamiento antropológico de Gabriel Marcel: un canto al ser humano, Pamplona, Eunsa, 2001.

Urabayen, J. Las raíces del humanismo de Emmanuel Levinas: el judaísmo y la fenomenología, Pamplona, Eunsa, 2005.

VV. AA., Nombres, Revista de Filosofía (Index), Universidad Nacional de Córdoba (Disponible en Internet: http://www.ffyh.unc.edu.ar/ dependencias/ciffyh/Nueva/Publicaciones_todas/Nombres.htm)

Vargas Guillén, G. "Excedencia y saturación. Fenomenología de la ausencia y la presencia de Dios", Anuario colombiano de fenomenología, 3, 2009, pp. 311-328. 
Vázquez Moro, U. El discurso sobre Dios en la obra de E. Lévinas, Madrid, Universidad Pontificia de Comillas, 1982.

Walton, R. “Reducción fenomenológica y figuras de la excedencia", Tópicos (Santa Fe), 16, 2008, 169-187.

Walton, R. “Apertura, desvelamiento y responsabilidad", El énfasis del infinito. Esbozos y perspectivas en torno al pensamiento de Emmanuel Lévinas (Bonzi, P. \& Fuentes, J. J., Eds.), Barcelona, Anthropos, 2009, pp. 51-75.

Walton, R. "La libertad como destino según Michel Henry: su despliegue y sentido", Anuario colombiano de fenomenología, 3, 2009, pp. 359-381. 